\title{
Evaluation of Real-World Healthcare Resource Utilization and Associated Costs in Children with Juvenile Idiopathic Arthritis: A Canadian Retrospective Cohort Study
}

\author{
Luiza R. Grazziotin (D) - Gillian Currie · Marinka Twilt • Maarten J. Ijzerman • Michelle M. A. Kip • \\ Hendrik Koffijberg · Susanne M. Benseler • Joost F. Swart • Sebastiaan J. Vastert • Nico M. Wulffraat • \\ Rae S. M. Yeung · Nicole Johnson · Nadia J. Luca · Paivi M. Miettunen · Heinrike Schmeling • \\ Deborah A. Marshall on behalf of the UCAN CAN-DU, UCAN CURE consortia
}

Received: May 2, 2021 / Accepted: June 3, 2021 / Published online: July 18, 2021

(c) The Author(s) 2021

\section{ABSTRACT}

Introduction: Juvenile idiopathic arthritis (JIA) is a chronic rheumatic disease, whose multifaceted care path can lead to significant

Supplementary Information The online version contains supplementary material available at https:// doi.org/10.1007/s40744-021-00331-X.

L. R. Grazziotin · G. Currie · D. A. Marshall ( $₫)$ Department of Community Health Sciences, Cumming School of Medicine, University of Calgary, Room 3C56, Health Research Innovation Centre, 3280 Hospital Drive NW, Calgary, AB T2N 4Z6, Canada

e-mail: damarsha@ucalgary.ca

L. R. Grazziotin · P. M. Miettunen - H. Schmeling D. A. Marshall

McCaig Institute for Bone and Joint Health, University of Calgary, Calgary, AB, Canada

L. R. Grazziotin · G. Currie · D. A. Marshall O'Brien Institute for Public Health, University of Calgary, Calgary, AB, Canada

L. R. Grazziotin · G. Currie · M. Twilt .

S. M. Benseler · N. Johnson · N. J. Luca .

H. Schmeling - D. A. Marshall

Alberta Children's Hospital Research Institute,

University of Calgary, Calgary, AB, Canada

G. Currie

Department of Paediatrics, Cumming School of

Medicine, University of Calgary, Calgary, AB,

Canada expenditure for the healthcare system. We aim to assess the real-world healthcare resource use (HCRU) and associated cost for children with JIA in a single center in Canada.

Methods: A single-center consecutive cohort of newly diagnosed patients with JIA attending the pediatric rheumatology clinic from 2011 to 2019 was identified using an administrative

M. Twilt · S. M. Benseler · N. Johnson · N. J. Luca - P. M. Miettunen - H. Schmeling Section of Rheumatology, Department of Paediatrics, Cumming School of Medicine, University of Calgary, Calgary, AB, Canada

M. J. Ijzerman · M. M. A. Kip · H. Koffijberg Department of Health Technology and Services Research, Faculty of Behavioural, Management and Social Sciences, Technical Medical Centre, University of Twente, Enschede, The Netherlands

S. M. Benseler Alberta Health Services, Calgary, AB, Canada

J. F. Swart · S. J. Vastert · N. M. Wulffraat Department of Pediatric Immunology and Rheumatology, Wilhelmina Children's Hospital / UMC Utrech, Utrecht, The Netherlands

J. F. Swart · S. J. Vastert - N. M. Wulffraat Faculty of Medicine, Utrecht University, Utrecht, The Netherlands

R. S. M. Yeung

Departments of Paediatrics, Immunology and Medical Science, The Hospital for Sick Children, University of Toronto, Toronto, Canada 
data algorithm and electronic medical charts. HCRU was estimated from six administrative health databases that included hospital admissions, emergency, outpatient care, practitioners' visits, medication, and laboratory and imaging tests. Costs were assigned using appropriate sources. We reported the yearly overall and JIAassociated HCRU and costs 5 years prior to and 6 years after the first visit to the pediatric rheumatologist. The Zhao and Tian estimator was used to calculate cumulative mean costs over a 6-year timeframe. Results were stratified by disease subtype.

Results: A total of 389 patients were identified. The yearly total overall mean costs per patient ranged between $\$ 804$ and $\$ 4460$ during the 5 years prior to the first visit to the pediatric rheumatologist and $\$ 8529$ and $\$ 10,651$ for the 6 years after. Medication cost, driven by use of biologic therapies, and outpatient visits were the greatest contributor to the total cost. The overall cumulative mean cost for 6 years of care was $\$ 48,649$ per patient, while the JIA-associated cumulative mean cost was $\$ 26,820$ per patient. During the first year of rheumatology care, systemic onset JIA had the highest cumulative mean overall cost, while oligoarticular JIA had the lowest cumulative mean cost.

Conclusion: The care pathway for children with JIA can be expensive, and complex-and varies by JIA subtype. Although the yearly total mean cost per patient was constant, the distribution of costs changes over time with the introduction of biologic therapies later in the care pathway. This study provides a better understanding of the JIA costs profile and can help inform future economic studies.

Keywords: Juvenile idiopathic arthritis; Costs; Administrative health databases; Drugs

\section{Key Summary Points}

Juvenile idiopathic arthritis (JIA) is a chronic inflammatory disease that affects 1 in every 1000 Canadian children younger than 16 years of age. However, there is a lack of estimates of real-world healthcare resource utilization and recent healthcare costs associated with the care of children with JIA.

This study assessed the healthcare resource utilization and respective healthcare costs associated with the care of children with JIA in a single center in Canada over 11 years in JIA care, including 5 years before the first visit with the pediatric rheumatologist.

This study showed an approximate 6.5fold increase in yearly overall costs prior to rheumatology care in comparison with the first year of rheumatology care for overall costs. It was also observed that yearly overall and JIA-associated mean cost do not decrease over time in the 6-year framework assessed.

Biologic therapies and outpatient costs are the main contributors for the yearly total costs after the first visit to the pediatric rheumatologist.

\section{DIGITAL FEATURES}

This article is published with digital features, including a summary slide, to facilitate understanding of the article. To view digital features for this article go to https://doi.org/10.6084/ m9.figshare.14725263.

\section{INTRODUCTION}

Juvenile idiopathic arthritis (JIA) is a chronic inflammatory disease that affects 1 in every 1000 Canadian children younger than 16 years 
of age [1-3]. JIA is characterized by joint pain and swelling, due to inflammation of the synovial lining of the affected joints $[1,2]$. As a chronic condition, long-term health issues may result, such as irreversible joint damage (that may require joint replacement), growth abnormalities, osteoporosis, pain, and psychological distress $[4,5]$. All these problems can negatively affect the quality of life of children with JIA and their families, prolong treatment during adulthood, and are associated with increased morbidity and mortality [5-8].

The healthcare pathway for children with JIA commonly involves several healthcare services including regular specialists' visits, emergency visits, hospital admissions, and drug medical treatment which can result in significant healthcare system expenditures $[9,10]$. JIA is divided in seven different subtypes with differences regarding joint involvement, extra-articular manifestations, disease severity, and longterm outcomes. The JIA subtypes can influence the rate of use of healthcare services $[11,12]$.

A recent scoping review identified 20 papers measuring healthcare resource utilization (HCRU) and costs in JIA [13]. Among the studies that report HCRU or costs, only four were published in the past 5 years [14-17]. Most of the studies (17 out of 20 ) had a follow-up of 1 year or less and none of these studies report results for HCRU separately from costs [13]. There is only one published study that reported healthcare system costs in JIA in Canada. However, this study was published in 2007 and was limited to a 2-year time horizon [18].

The lack of estimates of real-world HCRU and recent healthcare costs associated with the care of children with JIA over time is an important gap in the literature. The main objective of this study was to assess the HCRU and healthcare costs over time associated with the care of children with JIA. We also aimed to assess differences in healthcare costs considering all distinct JIA subtypes.

\section{METHODS}

We conducted a retrospective cohort study of consecutive children diagnosed with JIA using electronic medical charts and provincial health administrative data from Calgary, Alberta, Canada. Ethics approval for the following research was granted by the Conjoint Health Research Ethics Board at the University of Calgary (REB 19-0471).

\section{Setting and Participants}

To identify a cohort of patients newly diagnosed with JIA, we used a two-step process: (1) application of an administrative case ascertainment validated in a Canadian province to identify patients being assessed for JIA after 2011 at the pediatric rheumatology clinic at Alberta Children's Hospital, and (2) use of electronic medical charts to confirm JIA diagnosis and to collect demographic information. This study targeted patients that first visited a pediatric rheumatologist after 2011, as that is the year electronic clinical charts were consistently implemented in Alberta. The Alberta Children's Hospital is a specialty academic center in Calgary, which annually cares for approximately 100,000 children from newborn to age 17 from across Alberta (population of 4.3 million). The pediatric rheumatology clinic located at Alberta Children's Hospital is one of the two pediatric rheumatology centers in the province of Alberta.

As the first step to identify this cohort, we used an administrative case ascertainment algorithm reported by Shiff et al. [19]. Among the algorithms evaluated in this paper, we chose the one with the highest sensitivity. This ascertainment algorithm used JIA-related international classification of disease (ICD) codes (714 and 720 for ICD-9-CM, and M05, M06, M08, and M45 for ICD-10-CA) applied to the administrative databases' hospital discharge abstract and medical claims. The inclusion criteria in this algorithm are defined as follows: (1) patients with one or more of selected ICD codes in hospital discharge abstract; or (2) patients with two or more of selected ICD codes by any provider ever, one or more days apart in medical claims. After applying the administrative case ascertainment algorithm, we used the National Ambulatory Care Reporting System database, 
which includes outpatient visits, to select patients that had at least one visit to the pediatric rheumatology clinic at Alberta Children's Hospital. The linkage between electronic medical charts and health administrative data was performed using patient Personal Health Numbers (PHNs), date of birth, and sex.

After yielding all eligible patients using the ascertainment algorithm, two reviewers (LG, $\mathrm{CR}$ ) independently screened the list of patients using electronic medical charts from the pediatric rheumatology clinic to assess whether the patients met the following inclusion criteria: (1) patients with confirmed diagnosis of JIA, (2) patients whose diagnosis of JIA was established after 2011, and (3) patients that had at least two full assessments at the pediatric rheumatology clinic. A pediatric rheumatologist (MT) was consulted to reach a decision in cases where the JIA diagnosis was unclear. Patients were excluded if their arthritis was considered secondary to another disease (e.g., Crohn's disease), or they were diagnosed out of Alberta.

\section{Timeframes}

The patient's first visit to the pediatric rheumatologist was defined as the index date in this study. The date of the end of the study observation period was March 20, 2020. The timeframe for this study was defined as 5 years prior to first visit to the pediatric rheumatologist to a maximum of 6 years after the pediatric rheumatologist visit. The timeframe of 6 years was chosen with the aim of having at least $20 \%$ of sample when performing the analysis. For the subgroup analysis, the timeframe was reduced to 4 years because of the decrease of sample size in each subgroup after stratification by JIA subtype. For each JIA subtype, we reported results up to the year in which at least five patients were present. Since this data analysis intended to assess HCRU and costs of JIA during childhood, all variables were limited to the day the patient turned 18 years old.

\section{Data Sources}

\section{Clinical Variables}

We used the electronic medical charts to extract the following variables: JIA subtype, date of first visit to the pediatric rheumatologist, censored status at end of the study (i.e., patient still attending the pediatric rheumatology clinic, transitioned to adult care, moved out of the province/country, discharged owing to improvement, or stopped attending clinic because of unknown reasons), and date of symptoms onset. The data collection form was piloted using a sample of 50 patients by two reviewers (LG and CR) who refined the form and checked for consistency of abstraction and reporting. Data extraction of clinical variables was manually performed by one investigator (LG). Study data were collected and managed using REDCap electronic data capture tools hosted at University of Calgary [20, 21].

\section{Healthcare Resource Utilization}

All legal residents of the province of Alberta are eligible for Alberta Health Care Insurance Plan coverage for medically necessary physician services, hospital visits and stays, and emergency visits. A number of linkable provincial administrative datasets are available and contain healthcare encounters that can be assessed for research on health service utilization, including practitioner visits (i.e., general physicians, specialists, and non-medical practitioners such as optometrists, podiatrists, podiatric surgeons, and dentists), inpatient hospitalizations, emergency visits, outpatient visits, information on dispensed medication in outpatient and community pharmacies, and laboratory and diagnostic imaging tests [22]. More information on the datasets is available in Table S1 in the electronic supplementary material.

\section{Cost}

The valuation of HCRU in monetary units used multiple cost sources. We used the most updated costs available for each source, reported in Canadian dollars. Subsequently, all costs were adjusted to 2019 for consistency using consumer price index for healthcare in Alberta [23]. 
For hospital admissions, we multiplied the cost of a standard hospital stay in Alberta (2018-2019) by the resource intensity weight values available for each hospital admission. The resource intensity weight provides a measure of the relative resource consumption for a hospital visit compared to an average typical inpatient visit. For outpatient and emergency visits, we used the Comprehensive Ambulatory Care Classification System (CACS) grouper for fiscal year 2017/2018 provided by Alberta Health. The CACS grouper-assigned costs include functional center direct costs (e.g., nursing salary, salaries for patient care managers, medical staff administration, diagnostic imaging, laboratory tests) and functional center indirect costs (e.g., general administrative and support services, such as finance, material management, facilities management) [24]. For outpatient and emergency visits occurring prior to 2009, we used average overall CACS for emergency and non-emergency visits, since CACS grouper costs were not available prior to that time. Individual physician billing costs for visits were available from Alberta Schedule of Medical Benefits and were defined as the assessment amount for the service [25].

The unit costs for dispensed medications were obtained from 2020 provincial drug price lists. The costs of outpatient intravenous medication administration, including nursing and administrative costs, are accounted for in the outpatient costs. The Drug Identification Number (DIN) was used to match the dispensed medication with the unit cost. If DINs were unavailable in the drug price lists, the unit cost from other provincial drug price lists were used as a proxy estimate. Dispensed compounds that were not assigned a DIN or for which a unit cost could not be identified were excluded from the cost analysis.

Frequencies of laboratory and diagnostic imaging tests are described in the HCRU analysis. However, unit costs were not assigned because laboratory and diagnostic imaging tests are already accounted for in the inpatient grouper's costs (cost of a standard hospital stay) and outpatient and emergency grouper's costs (CACS grouper), which would otherwise lead to potential double-counting of costs.

\section{Overall and JIA-Associated HCRU and Costs}

In this study, we assessed the overall HCRU and costs, which include all resources used during the observational period evaluated in this study. We also evaluated JIA-associated HCRU and costs, where JIA was the main reason for the use of a determined resource. JIA-associated HCRU was defined as an inpatient hospitalization, outpatient visit, and practitioners' visit containing a JIA-related code (ICD-9-CM: 714 and 720, and ICD-10-CA: M05, M06, M08, and M45) as the main reason for the visit or admission. Dispensed medication associated with JIA treatment included non-steroidal anti-inflammatory drugs (NSAIDs), corticosteroids, synthetic disease-modifying anti-rheumatic drugs (sDMARDs), and biologic disease-modifying anti-rheumatic drugs (bDMARDs). These medications were identified in consultation with two pediatric rheumatologists (SB and MT). Laboratory and diagnostic imaging tests do not have an ICD code associated with their reporting; therefore, these were described only in the overall HCRU analysis. All joint injections performed were considered part of JIA-associated care.

\section{Statistical Analyses}

To investigate HCRU and costs, a descriptive analysis was conducted. Baseline characteristics of the participants were reported using descriptive summary measures, such as proportion, mean, standard deviation, median, minimum, and maximum. The reporting of HCRU was displayed in eight categories: hospital admissions, emergency visits, outpatient visits, total practitioners' visits (subdivided into general practitioner (GP) visits, specialist visits, and non-medical visits), medications dispensed (subdivided into bDMARDs, sDMARDs, and other medication), laboratory tests, diagnostic imaging tests, and joint injections. The reporting of costs was displayed in five categories: hospital admissions, emergency, outpatient care, total practitioners (subdivided into GP costs, specialist costs, and non-medical costs) and medications costs (subdivided into bDMARDs, sDMARDs, and other medication). 
Laboratory and diagnostic imaging tests (including joint injections) were not reported in a separate cost category because they are already accounted for in the inpatient, outpatient, and emergency costs (as mentioned previously).

To account for varying observation times among patients in the cohort and to analyze changes over time, HCRU and costs were summarized in two distinct ways. First, the mean HCRU and respective costs for each of the categories were calculated for specific time points: yearly for each of the 5 years prior to the first visit in the pediatric rheumatology clinic (index date) and yearly after that, up to 6 years after the first visit. The mean cost for each year included only patients with complete observation for the year of interest. We reported as mean, standard deviation, and range. For the assessment of sample size in the analysis prior to the first visit at the pediatric rheumatology clinic, we checked the date of birth and provincial residency registration to ensure patients were only included in the calculation of mean costs after they were born and if they

Table 1 Patient characteristics

\begin{tabular}{lll}
\hline Variables & $n$ & $\%$ \\
\hline Sex $(n=389)$ & 232 & 59.6 \\
Female & & \\
Disease subtype $(n=389)$ & 142 & 36.5 \\
Oligoarticular JIA & 139 & 35.7 \\
Polyarticular JIA & 69 & 17.7 \\
ERA & 16 & 4.1 \\
Undifferentiated JIA & 13 & 3.3 \\
Psoriatic JIA & 10 & 2.6 \\
Systemic JIA & & \\
\hline
\end{tabular}

Patient status by end of study $(n=389)$

$\begin{array}{lll}\text { Still attending PR clinic } & 214 & 55.0\end{array}$

$\begin{array}{lll}\text { Transitioned to adult care } & 114 & 29.3\end{array}$

Stopped attending PR clinic (unknown reason) $\quad 37 \quad 9.5$

$\begin{array}{lll}\text { Moved to another province or country } & 13 & 3.3\end{array}$

$\begin{array}{lll}\text { Discharged owing to JIA improvement } & 11 & 2.8\end{array}$

\begin{tabular}{llc}
\hline & Mean (SD) & Median (range) \\
\hline Age (years) at first visit with PR $(n=389)$ & $10.1(4.6)$ & $11(1-17)$ \\
Time (years) from symptom onset to first visit $(n=383)^{\mathrm{a}}$ & $0.96(1.2)$ & $0.45(0-11.4)$ \\
Years of observation $(n=389)$ & $4.0(2.2)$ & $3.6(0.1-9.6)$ \\
\hline
\end{tabular}

$J I A$ juvenile idiopathic arthritis, $E R A$ enthesis-related arthritis, $P R$ pediatric rheumatology, $S D$ standard deviation

a Six patients did not have information on time from symptom onset reported 


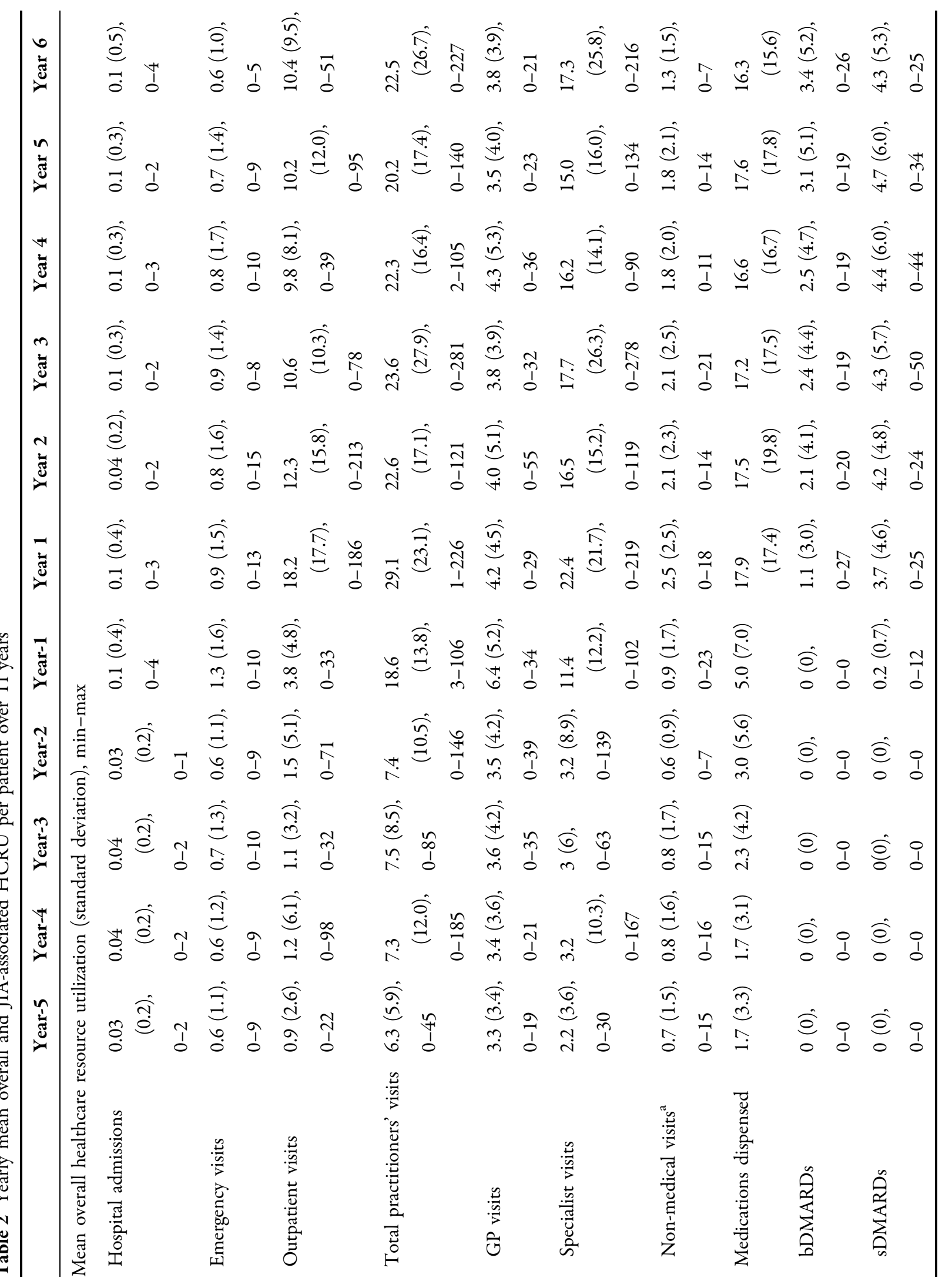




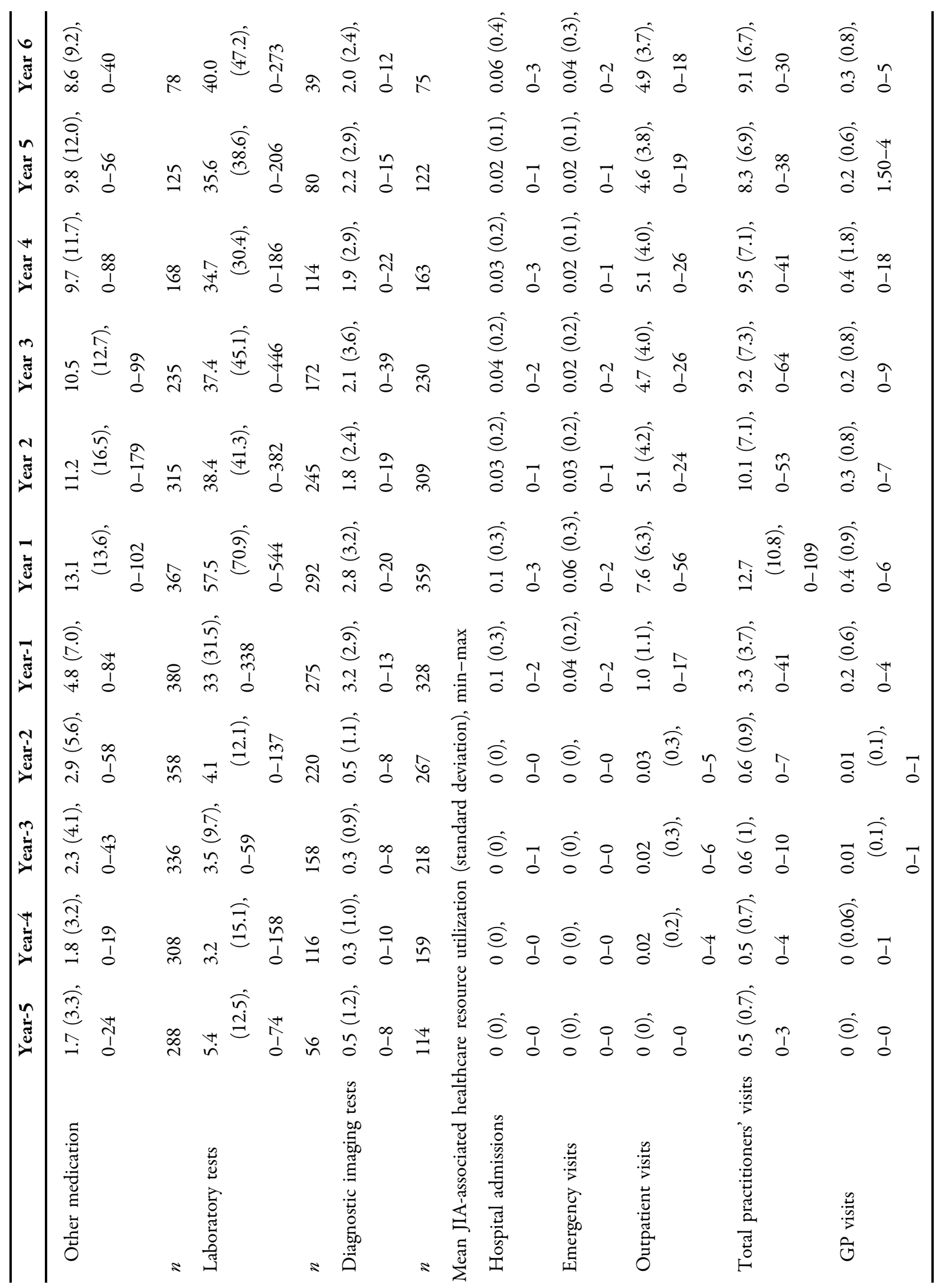




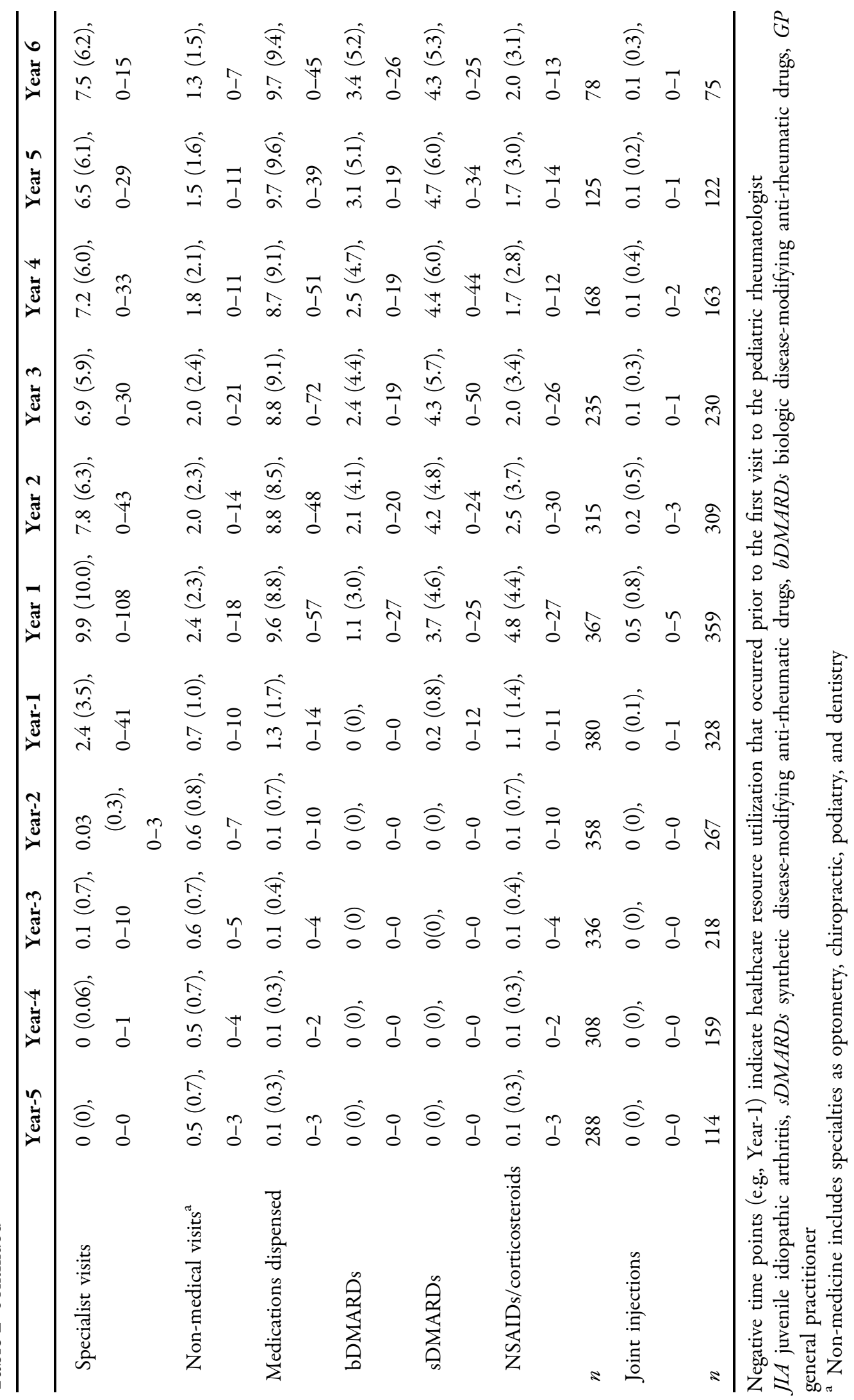




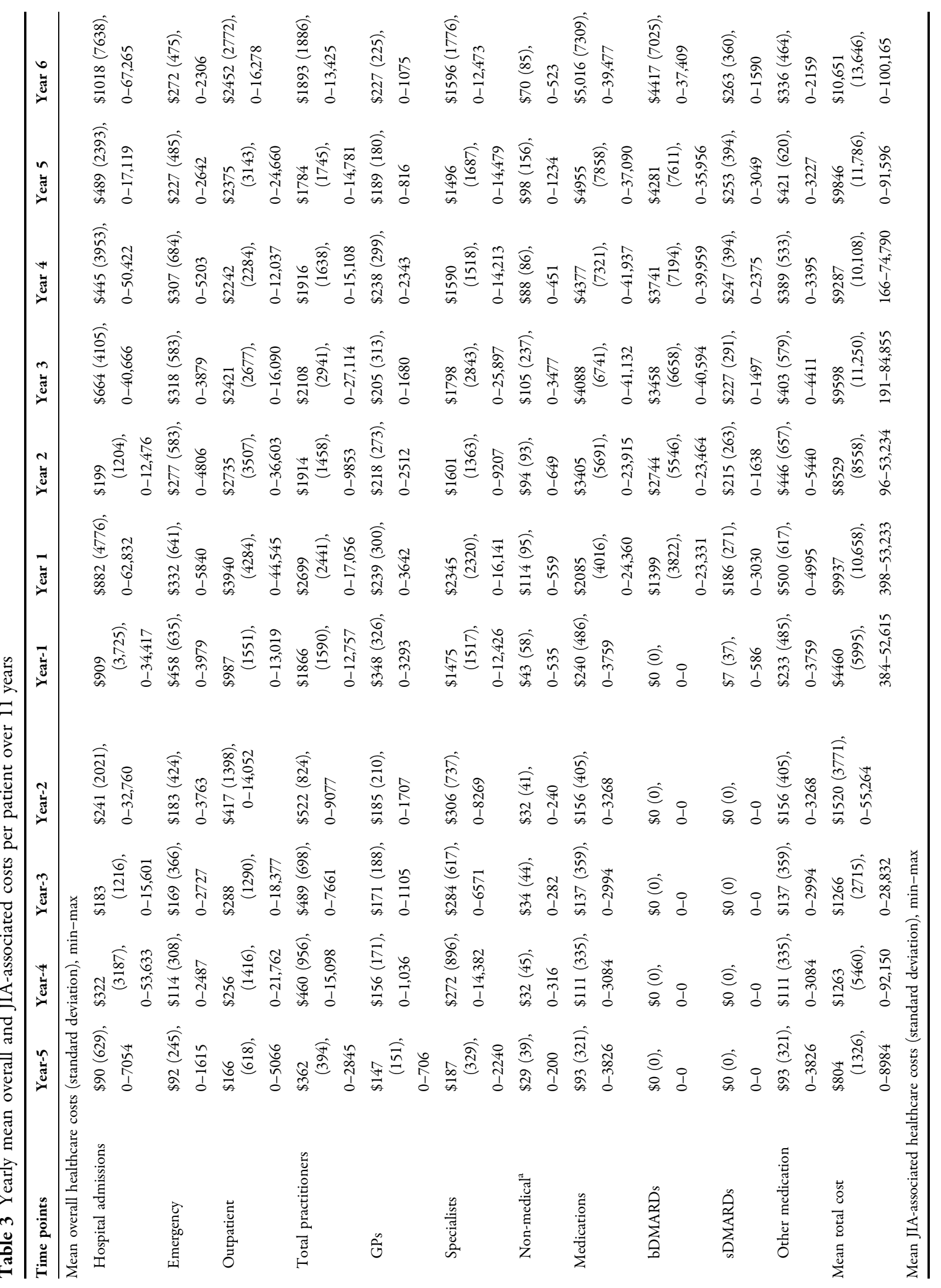




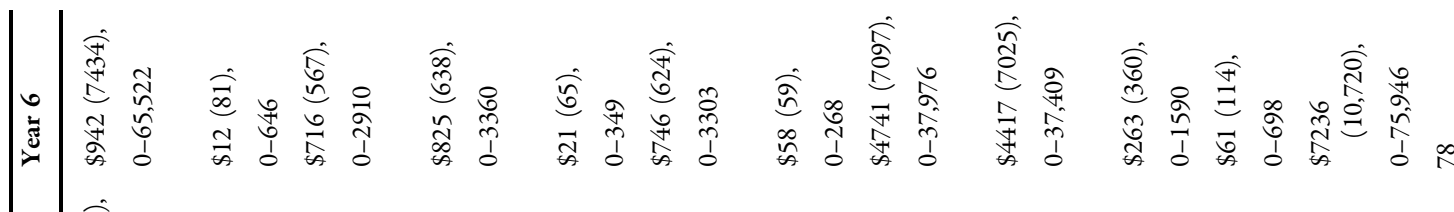

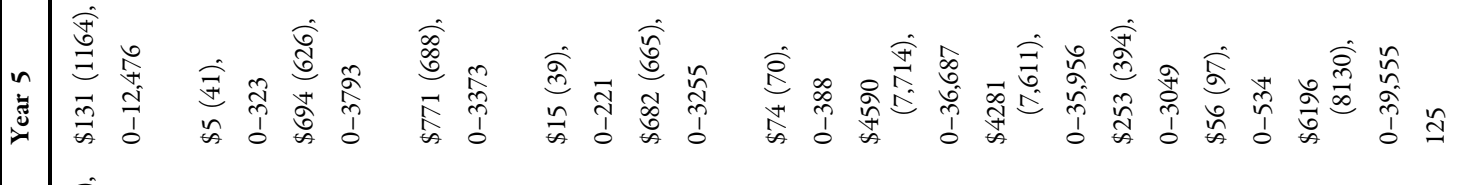

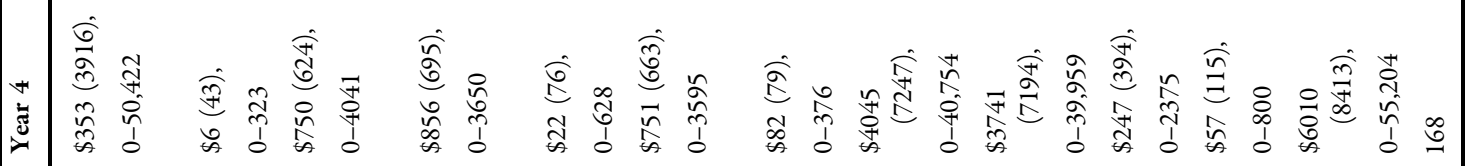

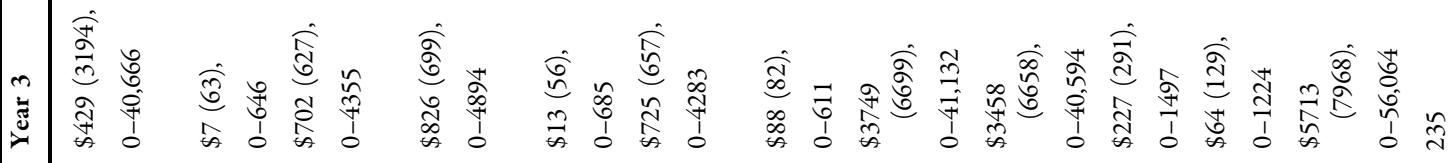

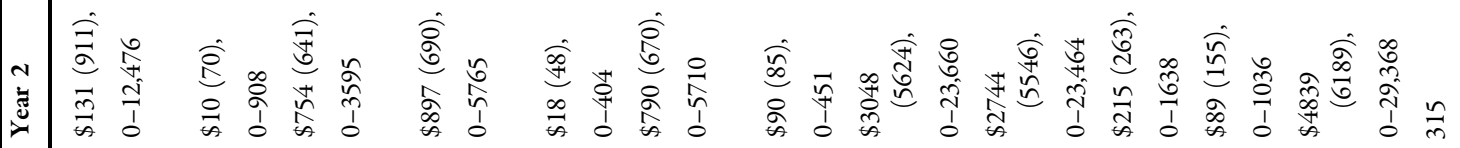

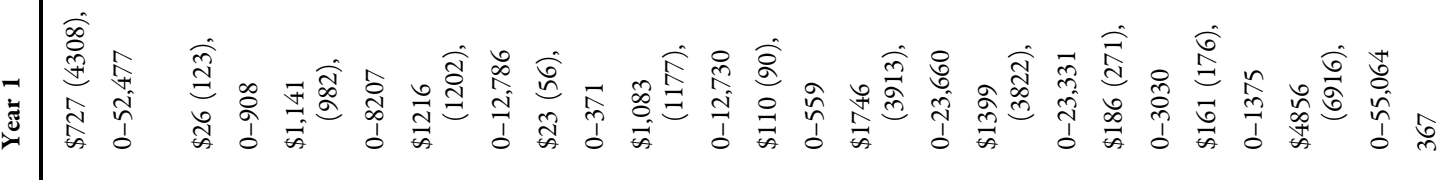

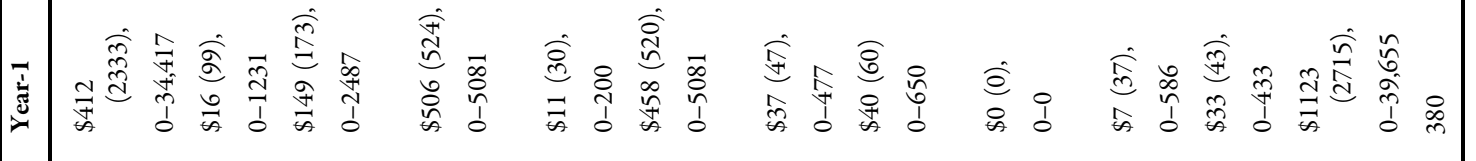

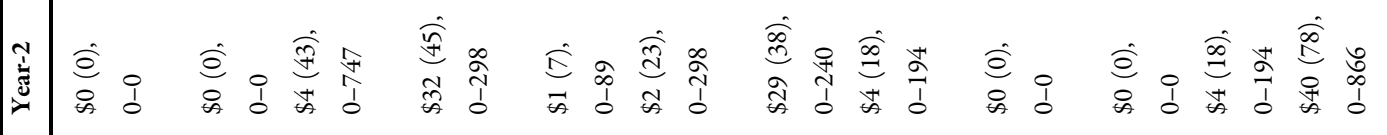

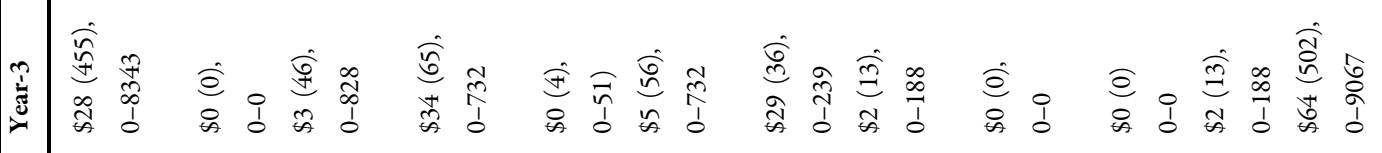

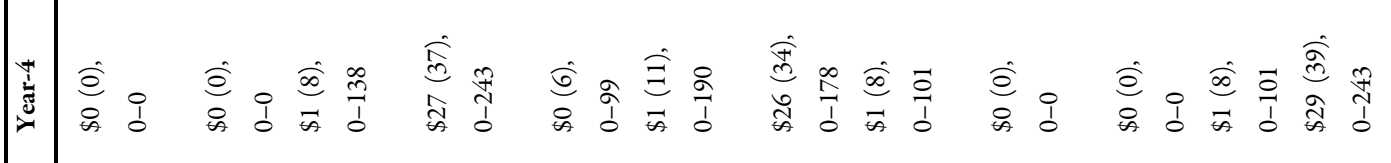

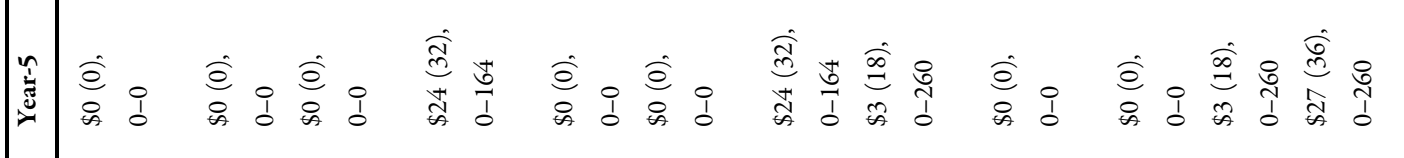

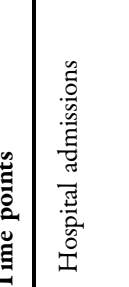
1

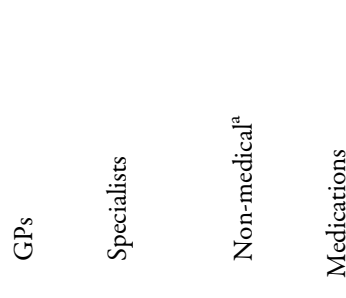

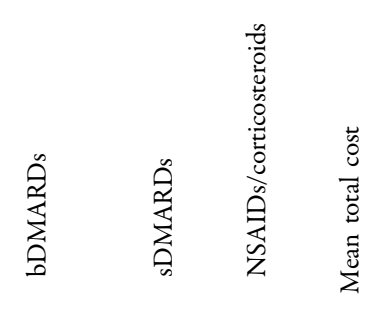

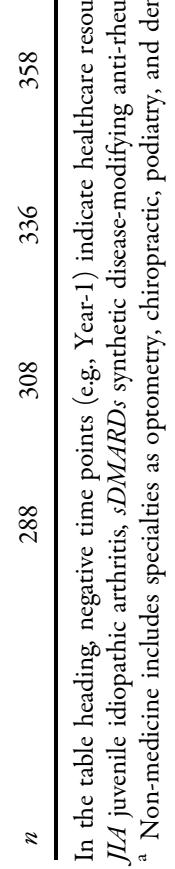


were residents of Alberta at the time. The sample size available for calculating mean number of laboratory and imaging tests (including joint injections) differs from the other datasets because these two databases were only implemented in April 2012 and January 2011, respectively. Therefore, the number of patients with complete years of observations was reduced for the yearly mean HCRU analysis.

Patients who were still attending the pediatric rheumatology clinic by the end of the study observation period, moved to another province, or were lost to follow-up were considered right censored in the analysis. To account for censoring in the healthcare cost estimates, we used the Zhao and Tian estimator (or weighted available sample estimator) for censored data with cost history [26]. The total cumulative mean cost including 95\% confidence intervals was calculated for overall and JIA-associated costs for a period of 6 years. The cumulative mean costs using the Zhao and Tian estimator are calculated by weighting the costs of the whole available sample, including censored cases, and partitioning the time over the observation period. The Zhao and Tian estimator has been shown to be more accurate than other estimators when cost history is available $[26,27]$.

We also stratified the total cumulative mean costs by six distinct JIA subtypes (i.e., oligoarticular JIA, polyarticular JIA, enthesitis-related arthritis (ERA), undifferentiated JIA, psoriatic JIA, and systemic JIA) and reported these results for a period of 4 years because of the decrease of sample size in each subgroup. In this subanalysis, as a result of the smaller sample size, we used bootstrap analysis (2000 iterations) to calculate $95 \%$ confidence interval.

All data analyses were performed using $\mathrm{R}$ (tidyverse and ccostr packages).

\section{RESULTS}

A total of 389 children with JIA met the inclusion criteria and were included in this analysis. Fewer than $0.8 \%$ of entries of dispensed medications could not be costed because of lack of DIN code or available unit cost.

\section{Clinical and Demographic Characteristics of the Cohort}

Most children in the cohort diagnosed with JIA were female $(60 \%)$ (Table 1$)$. Oligoarticular and polyarticular JIA (including rheumatoid factor positive and negative) were the most common subtypes, each accounting for $36 \%$ of the cohort. The least common subtype was systemic JIA $(n=10,2.6 \%)$. The mean age of first visit to the pediatric rheumatology clinic was 10.1 years (standard deviation 4.6). Most patients (55\%) were still attending the pediatric rheumatology clinic at the study end point, and the mean time recorded in the cohort was 4.0 years, ranging from 1.5 months to 9.6 years.

\section{Yearly Mean Overall and JIA-Associated HCRU and Costs}

The mean number of both overall and JIA-associated healthcare resources increased in the year prior to the pediatric rheumatologist visit, peaking in the first year of care in the rheumatology clinic, except for the emergency department and visits to the GP (Table 2). The mean number of emergency visits and that of GP visits are highest in the year prior to the visit to the pediatric rheumatologist.

The most frequent laboratory tests performed in the first year of care were complete blood count, C-reactive protein, and creatinine (see Table S2 in the electronic supplementary material for details). Anti-nuclear antibody (ANA) and rheumatoid factor (RF) testing was performed in $56 \%$ and $36 \%$ of patients, respectively, during the first year of care. This proportion increases to $79 \%(n=306 / 389)$ for ANA and $77 \%(n=298 / 389)$ for RF when the complete study timeframe is considered. Regarding imaging tests, $55 \%$ and $46 \%$ of patients had at least one general radiography and magnetic resonance imaging performed in the first year of care, respectively (see Table S3 in the electronic supplementary material for details).

The yearly total overall mean costs ranged between $\$ 804$ and $\$ 1520$ per patient during the 2-5 years prior to the first visit to the pediatric rheumatologist (Table 3). We observed an 


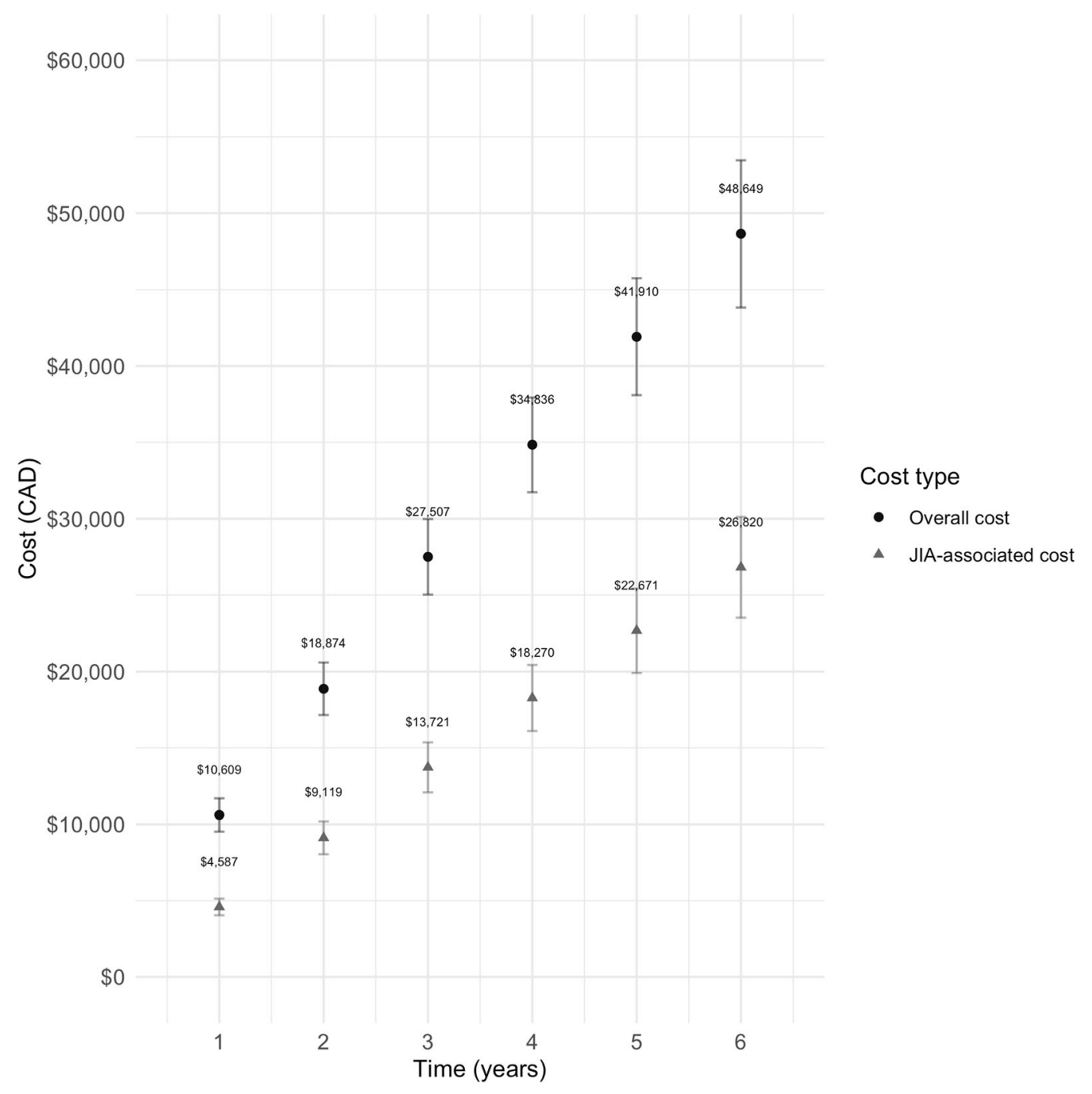

Fig. 1 Cumulative mean overall and JIA-associated costs per patient for a period of 6 years

approximately threefold increase in yearly costs from the second (Year-2, yearly mean cost of $\$ 1520$ ) to first year prior to the first visit to the pediatric rheumatologist (Year-1, yearly mean cost of $\$ 4460)$. In addition, a 6.5 -fold increase in yearly costs from the second year prior to the pediatric rheumatologist visit (Year-2, yearly mean cost $\$ 1520$ ) to the first year of care (year 1 , yearly mean cost \$9937) was noticed. The total overall mean costs per patient per year after the first visit to the pediatric rheumatologist remains in the range between $\$ 8529$ and $\$ 10,651$. The same pattern is observed for JIAassociated costs, showing an increase in costs in the year prior to the first visit to the pediatric rheumatologist and a range between $\$ 4839$ and $\$ 7236$ per patient per year during the first 6 years of care. Both overall and JIA-associated total mean costs remain fairly constant over the 6-year period after the first visit to the pediatric rheumatologist, with practitioners and outpatient care visits as the main cost drivers early on and then shifting with an increase in medication costs as a proportion of the total over time. Additionally, we evaluated the yearly mean costs of sDMARDs and bDMARDs stratified by JIA subtype for 4 years after the first visit to the pediatric rheumatologist. The yearly mean cost of sDMARDs ranged between $\$ 25$ and \$393. We observed that children with oligoarticular JIA 


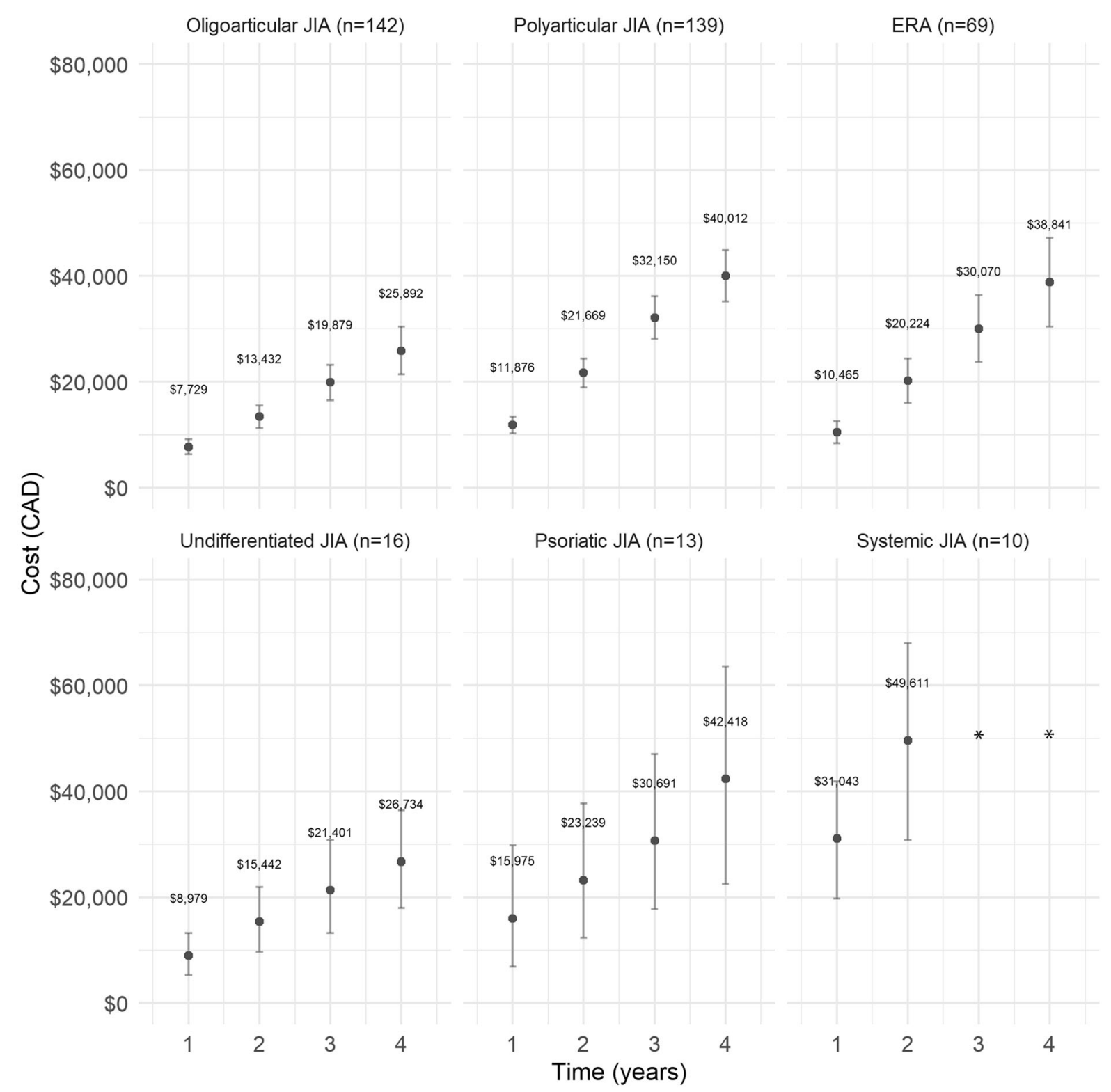

Fig. 2 Cumulative mean overall cost per patient for a period of 4 years stratified by subtype. ERA enthesis-related arthritis, * Not reportable, $n<6$

had the lowest yearly mean costs during the first year, while systemic JIA had the highest cost. When assessing costs specifically associated with use of bDMARDs, we observed that children with systemic JIA have the highest yearly mean costs for the first and second year after the first visit to the pediatric rheumatologist (third and fourth year were not assessed because of small sample size). Conversely, oligoarticular JIA is observed to have the lowest yearly mean cost across all 4 years (see the supplementary material Table S4).

\section{Cumulative Mean Overall and JIA- Associated Costs Over Period of 6 years}

The overall cumulative mean cost per patient for the 6 years of care was $\$ 48,649$ (95\% CI $\$ 43,833-53,465)$ per patient, while the JIA- 
associated cumulative mean cost was $\$ 26,820$ (95\% CI \$23,513-30,127) per patient (Fig. 1). The overall and JIA-associated cumulative costs by cost categories show the cumulative costs outpatient and practitioners visits decreasing over time after the first visit to the pediatric rheumatologist, while medication costs show a consistent increase over time (see Fig. S1 in the electronic supplementary material for details).

\section{Cumulative Mean Cost Stratified by JIA Subtype Over Period of 4 years}

Regarding the assessment of overall cumulative mean costs stratified by JIA subtype, systemic JIA has a higher mean cost per patient $(\$ 31,034$, CI 95\% \$19,947-42,139) among all the subtypes during the first year of rheumatology care (Fig. 2). However, as a result of the small sample size $(n=10)$, the confidence interval is wide. The distribution of cumulative mean costs after bootstrapping is available in Fig. S2 in the electronic supplementary material. Oligoarticular JIA had the lowest cost per patient over time. The cumulative overall mean costs for each cost category show that medication and outpatient costs were the main contributors to total overall cost in all subtypes (see Fig. S3 in the electronic supplementary material for details). A comparison between the JIA subtypes with larger sample sizes (polyarticular JIA, oligoarticular JIA, and ERA) showed that oligoarticular JIA has lower outpatient and medication costs after the second year of care when compared to polyarticular JIA and ERA. The three subtypes have similar costs regarding emergency, hospital admissions, and practitioner costs (see Fig. S4 in the electronic supplementary material for details).

\section{DISCUSSION}

The care pathway for children diagnosed with JIA can be expensive, long, and complex. Our study assessed the HCRU and respective healthcare costs over time associated with the care of children with JIA in a single center in Canada. This study is the first to assess a time window of 11 years in JIA care, including 5 years before the first visit with the pediatric rheumatologist, and report on recent frequency and costs of HCRU.

The advantage of assessing the HCRU and costs for the cohort prior to rheumatology care is that it established a baseline comparison for HCRU and costs post visit to the pediatric rheumatologist. The yearly mean overall cost per patient observed in the years prior to the first visit to the pediatric rheumatologist (ranging between $\$ 803$ and $\$ 1520$ per patient per year) were similar to the mean cost for children from the general population $(\$ 1181, n=1164$ ) reported in another recent Canadian study using comparable methodologies [28].

One of our main findings is that we observed an approximate 6.5-fold increase in yearly overall costs prior to rheumatology care in comparison with the first year of rheumatology care for overall costs. In the context of other pediatric chronic diseases, the total overall mean cost for children with JIA during the first year of rheumatology care exceeds that observed in children with asthma and diabetes, despite being lower than the costs associated with children with rare diseases [28].

Another important finding was that overall and JIA-associated total mean costs do not decrease over time after the first visit to the pediatric rheumatology clinic in the 6-year framework assessed. However, the distribution of costs changes, which is explained by the introduction of bDMARDs later in the care pathway. The costs of medication, particularly bDMARDs, together with outpatient and specialist costs, are the main factors driving the overall and JIA-associated total mean costs per year.

In addition to these findings, we identified that mean number of GP visits increases in the year prior to the first visit to the pediatric rheumatologist, which is consistent with the fact that patients are expected to be assessed by their GPs as JIA onset occurs (on average 6 months prior to the first visit to the pediatric rheumatologist). This same pattern is observed for the mean number of emergency visits, which is consistent with the higher likelihood of seeking emergency care before establishing 
ongoing care with the pediatric rheumatologist and with a defined treatment regimen for JIA.

In this study, in addition to reporting the yearly mean cost for patients with a full year of observation, we also reported the cumulative mean cost per patient over 6 years. This first analysis represents a snapshot of yearly mean costs for multiple years, while the cumulative mean cost shows the sum of these costs over time, to provide a broad understanding of the expenditure impact of JIA. Calculating the cumulative mean cost using the Zhao and Tian estimator has the advantage of using all data available, while accounting for censored cases and skewed cost data over time.

Our analysis showed that overall cumulative mean costs differ among JIA subtypes. These differences were more easily observed when comparing JIA subtypes with larger sample sizes, particularly that oligoarticular JIA has lower outpatient and medication costs compared to polyarticular JIA and ERA. These findings are consistent with disease severity, as patients with oligoarticular JIA are less likely to be treated with bDMARDs and have often a mild presentation of the disease [29]. We also observed that children with systemic JIA have a large yearly mean cost associated with use of bDMARDs, which is consistent with another study in the literature [9]. These findings are explained by the high proportion of patients with systemic JIA receiving bDMARDs (78\%) and the use of expensive treatment such as anakinra and tocilizumab.

Only one study describing healthcare costs in a cohort of patients with JIA in Canada was identified in the literature. This paper, published in 2007 by Bernatsky and colleagues, included 155 patients with JIA from two referral pediatric centers in Canada [18]. The paper collected cost data from surveys and reports costs standardized to annual mean per patient of \$3002 (95\% CI \$2330-3672) 2005 Canadian dollars (equivalent to $\$ 3656$ in 2019 Canadian dollars [23]). Our findings show a higher yearly total overall cost. One of the drivers of these differences could be that our study used electronic medical chart and health administrative databases to estimate HCRU. The use of medical records and health administrative datasets is considered a reliable source of evidence, particularly when evaluating long time horizons, since they can avoid recall bias [30]. Bernatsky et al.'s results could also reflect a routine clinical practice and costs from more than 15 years ago, which are different than current clinical practice. However, it was not possible to confirm that hypothesis as HCRU frequency was not reported in the study.

Studies from other countries assessing JIA HCRU and costs were identified in a systematic review published in 2019. Since none of the studies reported HCRU separately, it is challenging to attribute the differences in costs to higher frequency of HCRU or higher unit cost. Since that systematic review, another cost analysis study conducted in a cohort of Dutch patients with JIA was recently published [31]. We observed similar patterns between the Dutch cohort and our findings regarding constant cost over time, medication as the main driver of costs, and systemic JIA having the higher cost and oligoarticular JIA the lowest among all subtypes [31].

We expect that the findings regarding frequency of HCRU may be generalizable to other countries with accessibility of care similar to the Canadian healthcare system. In Canada, access to treatment with bDMARDs for patients with non-systemic JIA is generally granted for patients that failed sDMARDs as first-line therapy. Therefore, the timing and rate of medication cost increase will depend on countryspecific guidelines regarding types of therapies available and time to start those treatments. In Canada, many different types of bDMARDs (anti-tumor necrosis factor and interleukin-1 and -6 therapies) are approved by Heath Canada. However, access is different and dependent on many factors including province, patient insurance, and indication.

\section{Limitations}

Our study has limitations. First, we used an administrative case ascertainment algorithm to identify cases of JIA, which could lead to an incomplete capture of the population of patients diagnosed with JIA attending the 
pediatric rheumatology clinic in Calgary at that period. If that is the case, Shiff et al. indicated that the sensitivity of the algorithms decreases as the age of patients increases [19]. Therefore, patients not captured by the algorithm are more likely to be older than 11 years of age. However, we mitigate this issue by using the algorithm with the highest sensitivity (91.7\%) and by screening electronic medical charts. In addition, since JIA diagnosis was confirmed using electronic medical charts, the chances of including patients without JIA in this study are very low.

Another limitation of this study is related to the definition of JIA-associated HCRU and costs (defined as inpatient, outpatient, and practitioners' visits having a JIA code as the main reason for the encounter). This definition assumes that the codes can capture accurately the reasons for the visit. Many other nonspecific musculoskeletal codes can be used as the main reasons for these visits. We chose not to classify these non-specific codes as JIA-associated HCRU because it would generate greater uncertainty in our estimates. Therefore, our estimates of the JIA-associated HCRU and respective costs may be underestimated.

It is important to highlight that children with JIA may have variable HCRU and associated costs patterns, depending on disease severity and duration, risk factors, other clinical and genetic characteristics, and treatment type and onset. This study was not designed to directly compare alternative interventions, but rather to describe the HCRU and associated costs observed in routine clinical practice. Studies with larger sample sizes that would allow robust analysis are needed to evaluate the impact of these variables in the JIA care path.

Finally, we acknowledge that oligoarticular JIA can be categorized as extended or persistent, where oligoarticular-extended JIA might resemble polyarticular JIA RF negative in terms of medical pathway. However, most patients with oligoarticular JIA (84\%) were not differentiated between extended and persistent subtypes on the electronic medical charts. This issue could lead to biased estimation of oligoarticular JIA's HCRU and costs.

\section{CONCLUSION}

This study provides a unique perspective to understand the JIA trajectory by reporting the current JIA real-world HCRU and respective costs in routine clinical practice in Canada for the period before and after the first visit to the pediatric rheumatologist. We concluded that yearly overall and JIA-associated mean cost do not decrease over time in the 6-year framework assessed. The bDMARDs and outpatient costs are the main contributors for the yearly total costs after the first visit to the pediatric rheumatologist. This study reports current patterns of HCRU and costs in a large single-center cohort of patients with JIA over a longer time horizon than currently published in the literature, thereby capturing the economic implications of JIA over time, which can help inform future economic studies.

\section{ACKNOWLEDGEMENTS}

Funding. This work and the journal's Rapid Service Fee were supported by the Canadian Institutes for Health Research (Canada) [grant number 381280]; Genome Canada (Canada); ZonMW (the Netherlands); and the Reumafonds (the Netherlands). DAM is supported by the Arthur J.E. Child Chair in Rheumatology and a Canada Research Chair in Health Systems and Services Research (2008-2018). SB is supported by the Husky Energy Chair in Child and Maternal Health and the Alberta Children's Hospital Foundation Chair in Pediatric Research. RSMY is supported by the Hak-Ming and Deborah Chiu Chair in Paediatric Translational Research. LRG is supported by Alberta Innovates Graduate Studentship and Arthritis Society (TGP-18-0244).

Medical Writing, Editorial and other Assistance. We would like to acknowledge the contributions of Carolina de La Rosa (CR), a reviewer on the data extraction for the electronic medical charts. In addition, we would 
like to acknowledge Alberta Health Services for providing the data for the study.

Authorship. All named authors meet the International Committee of Medical Journal Editors (ICMJE) criteria for authorship for this article, take responsibility for the integrity of the work as a whole, and have given their approval for this version to be published.

Authors' Contributions. Luiza R. Grazziotin, Deborah A. Marshall, Gillian Currie, Marinka Twilt, Maarten J. Ijzerman, Michelle M.A. Kip were involved in the conception and design of the study. Deborah A. Marshall and Gillian Currie contributed to the acquisition of data. Luiza R. Grazziotin conducted the data analysis. All authors were involved in the interpretated the data. Luiza R. Grazziotin drafted the manuscript, and all other authors were major contributors in critically reviewing the manuscript. All authors read and approved the final manuscript.

Prior Presentation. This study was previously presented in the form of abstract during the 2021 Canadian Rheumatology Association Annual Scientific Meeting held online from February 24 to 26, 2021.

Disclosures. Deborah A. Marshall reports non-financial support from consultancy (Illumina) and ISPOR, and personal fees from Analytica, outside the submitted work; Rae S.M. Yeung reports consulting fees from Novartis and Lily outside the submitted work. Sebastiaan J. Vastert reports grants and personal fees from SOBI and Novartis during the conduct of the study; Joost F. Swart reports grants from SOBI, outside the submitted work. Luiza R. Grazziotin, Gillian Currie, Marinka Twilt, Maarten J. Ijzerman, Michelle M.A. Kip, Hendrik Koffijberg, Susanne M. Benseler, Nico M. Wulffraat, Nicole Johnson, Nadia J. Luca, Paivi M. Miettunen and Heinrike Schmeling have nothing to disclose. The authors have no other relevant affiliations or financial involvement with any organization or entity with a financial interest in or financial conflict with the subject matter or materials discussed in the manuscript apart from those disclosed.

Compliance with Ethics Guidelines. Ethics approval for the following research was granted by the Conjoint Health Research Ethics Board at the University of Calgary (REB 19-0471).

Data Availability. The data that support the findings of this study are available from Alberta Health Services, but restrictions apply to the availability of these data, which were used under license for the current study, and so are not publicly available. Data are however available from the authors upon reasonable request and with permission of Alberta Health Services.

Open Access. This article is licensed under a Creative Commons Attribution-NonCommercial 4.0 International License, which permits any non-commercial use, sharing, adaptation, distribution and reproduction in any medium or format, as long as you give appropriate credit to the original author(s) and the source, provide a link to the Creative Commons licence, and indicate if changes were made. The images or other third party material in this article are included in the article's Creative Commons licence, unless indicated otherwise in a credit line to the material. If material is not included in the article's Creative Commons licence and your intended use is not permitted by statutory regulation or exceeds the permitted use, you will need to obtain permission directly from the copyright holder. To view a copy of this licence, visit http://creativecommons.org/licenses/by$\mathrm{nc} / 4.0 /$.

\section{REFERENCES}

1. Prakken B, Albani S, Martini A. Juvenile idiopathic arthritis. Lancet. 2011;377(9783):2138-49.

2. Ravelli A, Martini A. Juvenile idiopathic arthritis. Lancet. 2007;369(9563):767-78.

3. Shiff NJ, Lix LM, Oen K, et al. Chronic inflammatory arthritis prevalence estimates for children and adolescents in three Canadian provinces. Rheumatol Int. 2015;35(2):345-50. 
4. Kulas DT, Schanberg L. Juvenile idiopathic arthritis. Curr Opin Rheumatol. 2001;13(5):392-8.

5. McCann LJ, Wedderburn LR, Hasson N. Juvenile idiopathic arthritis. Arch Dis Child. 2006;91(2): ep29-ep36.

6. Mullick MS, Nahar JS, Haq SA. Psychiatric morbidity, stressors, impact, and burden in juvenile idiopathic arthritis. J Health Popul Nutr. 2005;23(2): 142-9.

7. Thomas E, Symmons DP, Brewster DH, Black RJ, Macfarlane GJ. National study of cause-specific mortality in rheumatoid arthritis, juvenile chronic arthritis, and other rheumatic conditions: a 20 year followup study. J Rheumatol. 2003;30(5):958-65.

8. Grazziotin LR, Currie G, Kip MMA, et al. Health state utility values in juvenile idiopathic arthritis: what is the evidence? Pharmacoeconomics. 2020;38(9):913-26.

9. Kip MMA, de Roock S, Currie G, et al. Costs of medication use among patients with juvenile idiopathic arthritis in the Dutch healthcare system. Expert Rev Pharmacoecon Outcomes Res. 2020;1-10. https://doi.org/10.1080/14737167. 2021.1857241 .

10. Prince FH, de Bekker-Grob EW, Twilt M, et al. An analysis of the costs and treatment success of etanercept in juvenile idiopathic arthritis: results from the Dutch Arthritis and Biologicals in Children register. Rheumatology. 2011;50(6):1131-6.

11. Yucel IK, Seyahi E, Kasapcopur O, Arisoy N. Economic impact of juvenile idiopathic arthritis and familial Mediterranean fever. Rheumatol Int. 2012;32(7):1955-62.

12. Gidman W, Meacock R, Symmons D. The humanistic and economic burden of juvenile idiopathic arthritis in the era of biologic medication. Curr Rheumatol Rep. 2015;17(5):31.

13. Kip MMA, Currie G, Marshall DA, et al. Seeking the state of the art in standardized measurement of health care resource use and costs in juvenile idiopathic arthritis: a scoping review. Pediatr Rheumatol Online J. 2019;17(1):20.

14. Kuhlmann A, Schmidt T, Treskova M, et al. Social/ economic costs and health-related quality of life in patients with juvenile idiopathic arthritis in Europe. Eur J Health Econ. 2016;17:79-87.

15. Luca NJ, Burnett HF, Ungar WJ, et al. Cost-effectiveness analysis of first-line treatment with biologic agents in polyarticular juvenile idiopathic arthritis. Arthritis Care Res. 2016;68(12):1803-11.
16. Shepherd J, Cooper K, Harris P, Picot J, Rose M. The clinical effectiveness and cost-effectiveness of abatacept, adalimumab, etanercept and tocilizumab for treating juvenile idiopathic arthritis: a systematic review and economic evaluation. Health Technol Assess. 2016;20(34):1-222.

17. Shenoi S, Horneff G, Cidon M, et al. The burden of systemic juvenile idiopathic arthritis for patients and caregivers: an international survey and retrospective chart review. Clin Exp Rheumatol. 2018;36(5):920-8.

18. Bernatsky S, Duffy C, Malleson P, Feldman DE, St Pierre Y, Clarke AE. Economic impact of juvenile idiopathic arthritis. Arthritis Rheum. 2007;57(1): $44-8$.

19. Shiff NJ, Oen K, Rabbani R, Lix LM. Validation of administrative case ascertainment algorithms for chronic childhood arthritis in Manitoba. Canada Rheumatol Int. 2017;37(9):1575-84.

20. Harris PA, Taylor R, Thielke R, Payne J, Gonzalez N, Conde JG. Research electronic data capture (REDCap) - a metadata-driven methodology and workflow process for providing translational research informatics support. J Biomed Inf. 2009;42(2): 377-81.

21. Harris PA, Taylor R, Minor BL, et al. The REDCap consortium: building an international community of software platform partners. J Biomed Inf. 2019;95:103208.

22. Alberta Health, Health Analytics Branch, Overview of Administrative Health Datasets. 2017. https:// open.alberta.ca/dataset/657ed26d-eb2c-4432-b9cb0ca2158f165d/resource/38f47433-b33d-4d1e-b959df312e9d9855/download/research-health-datasets. pdf. Accessed 5 Dec 2020.

23. Statistics Canada. Table 18-10-0004-08, Consumer Price Index, monthly, percentage change, not seasonally adjusted, Canada, provinces, Whitehorse and Yellowknife - Health and personal care. https://doi.org/10.25318/1810000401-eng. Accessed 22 Dec 2020.

24. Alberta Health, Health Analytics Branch. Interactive Health Data Application - Hospital Ambulatory Care Case Costing 2015. http://www.ahw.gov.ab. ca/IHDA_Retrieval/ShowMetaDataNotesServlet?15 04. Accessed 8 Oct 2020.

25. Alberta Health Care Insurance Plan - Medical Price List: Alberta Government; 2020. https://open. alberta.ca/dataset/568f8505-2304-4ce2-882c-2bbbc 314b739/resource/94892cea-00e5-4a2b-9633-4216 3def7ba6/download/health-somb-medical-price-list -2020-05.pdf. Accessed 8 Oct 2020. 
26. Børty L, Brøndum R, Bøgsted M. ccostr: an R package for estimating mean costs with censored data. J Open Source Softw. 2019;4(41):1593.

27. Chen S, Rolfes J, Zhao H. Estimation of mean health care costs and incremental cost-effectiveness ratios with possibly censored data. Stand Genomic Sci. 2015;15(3):698-711.

28. Marshall DA, Benchimol EI, MacKenzie A, et al. Direct health-care costs for children diagnosed with genetic diseases are significantly higher than for children with other chronic diseases. Genet Med. 2019;21(5):1049-57.
29. Giancane G, Consolaro A, Lanni S, Davì S, Schiappapietra B, Ravelli A. Juvenile idiopathic arthritis: diagnosis and treatment. Rheumatol Ther. 2016;3(2):187-207.

30. Evans C, Crawford B. Patient self-reports in pharmacoeconomic studies. Their use and impact on study validity. Pharmacoeconomics. 1999;15(3): 241-56.

31. van den Berg I, Kip MMA, Marshall DA, et al. Juvenile idiopathic arthritis is associated with considerable financial burden to society: results of a Dutch cost analysis. Value Health. 2019;22:S632. 